\title{
Doubly-polarised pion photoproduction and the GDH sum rule on the nucleon at MAMI
}

\author{
Federico Cividini* on the behalf of the A2 Collaboration \\ Mainz University \\ E-mail: fecividi@uni-mainz.de
}

\author{
Susanna Costanza \\ Department of Physics, University of Pavia \\ INFN Pavia \\ E-mail: susanna.costanza@pv.infn.it
}

\begin{abstract}
New measurements of the helicity dependence of the total inclusive photo-absorption cross section and of the partial cross sections for several reaction channels on the proton and on the neutron were carried out at the tagged photon beam facility of the MAMI accelerator (Mainz) by the A2 experiment in the energy region $200<E_{\gamma}<1500 \mathrm{MeV}$. These new, high-quality doubly-polarized pion-photoproduction data sets give a valuable input to the study of the nucleon structure and excitation spectra of protons and neutrons, by providing a contribution to the partial wave analysis models and by allowing to constrain the multipole solution of the different analyses. Furthermore, the helicity dependent observables provide the main ingredient for the verification of the well-known Gerasimov-Drell-Hearn (GDH) sum rule, which relates the helicity-dependent photoabsorption process to the main static nucleon properties (magnetic moment, mass, charge, spin). For this reason, such a verification is of particular interest in the understanding of the nucleon spin structure, the $\gamma-N$ interaction, as well as the physics of strongly interacting systems. Thanks to the use of polarized deuteron and ${ }^{3} \mathrm{He}$ targets, the A2 experiment can study all the $\gamma N \rightarrow N \pi(\pi)$ partial channels, as well as the total cross sections, for the neutron too. The new precise results on double-polarization measurements of the total and differential cross sections for the partial $\gamma N \rightarrow \pi N$ channels on the proton and on the neutron, obtained by the A2 collaboration, are compared to the existing model predictions and to the few, available results. These new data are significantly increasing the available statistics, especially on the neutron, thus providing an important testing ground for all existing models. Moreover, the results obtained on ${ }^{3} \mathrm{He}$ give information not only on the GDH integral on the neutron, but also on the ${ }^{3} \mathrm{He}$ nuclear structure and allow an investigation of the nucleon properties inside this nucleus.
\end{abstract}

XVII International Conference on Hadron Spectroscopy and Structure

25-29 September, 2017

University of Salamanca, Salamanca, Spain

${ }^{*}$ Speaker. 


\section{Introduction}

The internal structure of the nucleon has been an important subject of intense study in nuclear and particle physics since many decades, both from the experimental and theoretical point of view. The excited states of the nucleon are related to the fundamental properties of the strong interaction, just like the excitation spectra of atoms reflect the properties of the electromagnetic interaction. For this reason the investigation of the excited states of the nucleon is a fundamental step towards the understanding of the nucleon structure. Photoproduction reactions of single pseudoscalar mesons from the nucleon is described theoretically by 4 helicity amplitudes, which lead to 16 real experimental observables. This is possible by exploiting the polarisation degrees of freedom of the incident photon, and along with spin degrees of freedom in the target and recoiling nucleons. The measurement of 7 (8) properly chosen observables is necessary to perform a multipole expansion analysis.

\section{Helicity dependence}

The double-polarisation observable E requires a circularly polarised photon beam and a longitudinally polarised target. It derives from the differential cross section of pseudo-scalar meson photoproduction;

$$
\left.\frac{d \sigma}{d \Omega}(\theta)=\frac{d \sigma}{d \Omega_{0}}(\theta)\left[1 \pm P_{z} P_{\odot} E\right)\right]
$$

It is possible to extract the $\mathrm{E}$ observable from eq. 2.1 in the following way:

$$
\left.N\right|_{ \pm \alpha} ^{ \pm P_{z}}(\theta)=N(\theta)\left[1 \pm P_{\odot} P_{z} E\right] \rightarrow E=\frac{\sigma^{1 / 2}-\sigma^{3 / 2}}{\sigma^{1 / 2}+\sigma^{3 / 2}}=\frac{N_{\uparrow \downarrow}-N_{\uparrow}}{N_{\uparrow \downarrow}+N_{\uparrow}} \cdot \frac{1}{P_{\odot}} \cdot \frac{1}{P_{z}},
$$

where $N_{\uparrow \downarrow}$ and $N_{\uparrow}$ are the helicity-dependent events, $P_{z}$ and $P_{\odot}$ are the degree of target and beam polarisation.

The GDH sum rule was proposed by Gerasimov-Drell-Hearn in 1966 [4, 5]. It is derived from some very general fundamental physical principles, like Lorentz invariance, analyticity and unitarity. It connects the nucleon anomalous magnetic moment (AMM) $\kappa$, the spin $S$ and the mass $M$ of a nucleon with the integral over the weighted helicity difference of the total absorption cross section for circularly polarised photons on a longitudinally polarised nucleon target:

$$
I_{G D H}=\int_{v_{t h}}^{\infty} \frac{\sigma_{\uparrow}-\sigma_{\uparrow \downarrow}}{v} d v=4 \pi^{2} \kappa^{2} \frac{e^{2}}{M^{2}} S
$$

where $v$ is the photon energy, and $\sigma_{\uparrow}$ and $\sigma_{\uparrow \downarrow}$ the total absorption cross section for parallel and antiparallel orientation of photon and particle spins. The lower limit of the integral, $v_{t h}$, is the pion photoproduction and photodisintegration threshold for a nucleonic and nuclear target.

The verification of the GDH sum rule is very interesting for verifyng the $\gamma$ nucleon interaction, as well as for the physics of strongly interacting systems and for the existing photo-reaction models. By studying the helicity dependent $N \pi$ channels, it is also possible to test multipole models, to access new observables and to study the baryonic resonances. Thanks to the use of polarised deuteron and ${ }^{3} \mathrm{He}$ targets, the $\mathrm{A} 2$ Collaboration can investigate all the $\gamma N \rightarrow N \pi(\pi)$ partial channels, as well 
as the total cross section, for the proton and the neutron. It can also give a better insight into the GDH sum rule and perform an accurate investigation of the baryonic resonance properties.

\section{A2 experimental setup @ MAMI}

The experimental setup of the A2 Collaboration is located in the tagger-hall at the MAMI beam facility in Mainz (Germany). The MAMI accelerator provides a polarised electron beam of 1557 $\mathrm{MeV}$ with a polarisation up to $\sim 75-78 \%$. The photon beam is produced via bremsstrahlung and the energy of the single photons is tagged using the Glasgow-Mainz tagger spectrometer with an energy resolution of $\sim 2 \mathrm{MeV}$. Several targets can be used in the A2 apparatus. For the experiments described in this paper, a frozen-spin deuterated butanol target $\left(\mathrm{C}_{4} \mathrm{DOD}_{9}\right)$, and a high pressure ${ }^{3} \mathrm{He}$ target have been used. The deuterated butanol target consists in a $2 \mathrm{~cm}$ long cell which is located inside a cryostat that provides a temperature of $\sim 25 \mathrm{mK}$ using a dilution refrigerator mixture of ${ }^{3} \mathrm{He} /{ }^{4} \mathrm{He}$. The nucleons are polarised via Dynamic Nuclear Polarisation (DNP), using a Micro wave irradiation at $70 \mathrm{GHz}$ and $2.5 \mathrm{~T}$ up to an initial polarisation degree of $70 \%$. During the data taking, due to the large acceptance of the detector, it is not possible to keep the large magnet around the target. The target is then kept in a frozen spin mode, with a temperature of $25 \mathrm{mK}$ provided by the cryostat and, in combination with a magnetic field of $0.68 \mathrm{~T}$, it was possible to have a relaxation time of $\sim 1000$ hours. With this condition it is possible to run for one week with a high polarisation degree before the repolarisation is required. Since the deuterated butanol target has been used, the count rates include the contribution of both the polarisable quasi-free protons and neutrons in deuteron nuclei and the unpolarised bound nucleons in carbon and oxygen nuclei. The amount of events generated from the polarisable deuteron is specified by introducing in the eq. 2.2 the dilution factor $d$ :

$$
E=\frac{N_{\uparrow \downarrow}-N_{\uparrow}}{N_{\uparrow \downarrow}+N_{\uparrow}} \cdot \frac{1}{P_{\odot}} \cdot \frac{1}{P_{z}} \cdot \frac{1}{d}
$$

it takes into account the number of polarised nuclei inside the target $\left(d=N_{\text {deuteron }} /\left(N_{\text {butanol }}\right)\right)$, which was extracted during a dedicated data taking with a carbon foam target. During this run also differences in acceptance, photon flux and target density during the carbon and butanol beamtimes are taken into account.

For the gaseous ${ }^{3} \mathrm{He}$ an initial polarisation value up to $70 \%$ was obtained, via the metastability exchange optical pumping (MEOP) method, with a total relaxation time of $\sim 100$ hours.

The target cell is located inside the central detector apparatus, and surrounded by the Particle IDentification (PID) detector, made of 24 plastic scintillators. Around the PID there are two MultiWire Proportional Chambers (MWPCs), which provide the discrimination between charged and neutral particles and track the coordinates of the charged particles. These detectors are enclosed inside the Crystal Ball (CB) calorimeter, which consists in $672 \mathrm{NaI}$ crystals covering the azimuthal $(\phi)$ region from $0^{\circ}$ to $360^{\circ}$ and the polar $(\theta)$ from $21^{\circ}$ to $159^{\circ}$. In the forward region, TAPS, a calorimeter of $366 \mathrm{BaF}_{2}$ and $72 \mathrm{PbWO}_{4}$ scintillators, covers the polar region between $1^{\circ}$ to $21^{\circ}$. The full setup provides precise energy and angle measurements, and particle identification in the $97 \%$ of the solid angle. 


\section{Results on deuteron}

Figure 1 shows the preliminary results of the helicity dependence of the total inclusive cross section $\Delta \sigma=\left(\sigma_{\uparrow \downarrow}-\sigma_{\uparrow}\right)$ on the deuteron. The results for the partial channels $\gamma d \rightarrow \pi^{0} X$ are depicted in Fig. 2. The A2 data (blue points) are compared with good agreement to the GDH Collaboration results (red points) [9], and the $\pi^{0}$ data is compared also with the sum from MAID model for free-proton and free-neutron [10]. In Fig. 2 there are 4 examples of helicity-dependence differential cross section for the partial channels $\gamma d \rightarrow \pi^{0} X$. The magenta line shows the sum from the MAID model for free-proton and free-neutron. After the identification of the proton or the neutron and the subtraction of the carbon and oxygen contribution, the deuterated butanol target allows also the extrapolation of the double-polarised observable E. In this way it is possible to expand the results from [11] at lower energy and on a larger angular coverage.

\section{Results on ${ }^{3} \mathrm{He}$}

Figure 3 shows the unpolarised and helicity-dependent total cross sections for the partial chan-
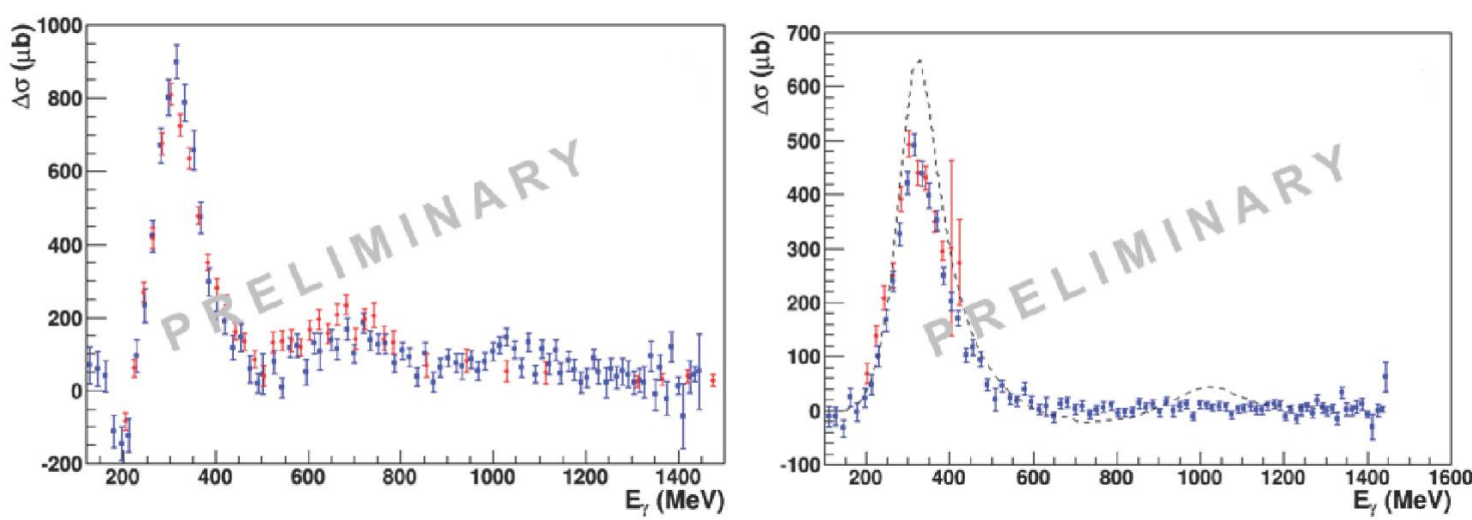

Figure 1: Results on deuteron, on the left the helicity dependent total inclusive cross section for the $\gamma d \rightarrow X$, on the right the helicity dependent total cross section for the semi-exclusive channels $\gamma d \rightarrow \pi^{0} X$. The A2 results (blue circles) are compared to the results from the GDH Collaboration (red circles) and to the sum of MAID for free proton and neutron (dashed line).
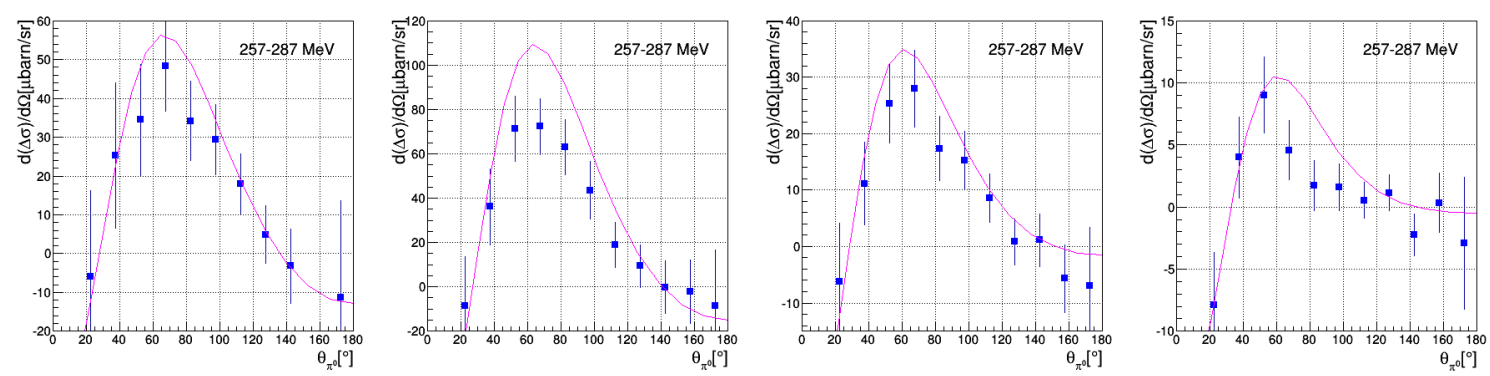

Figure 2: Results on deuteron, helicity dependent differential cross section for the semi-exclusive channels $\gamma d \rightarrow \pi^{0} X$. The A2 results (blue circles) are compared with the sum of the MAID model for free-proton and free-neutron (magenta line). 

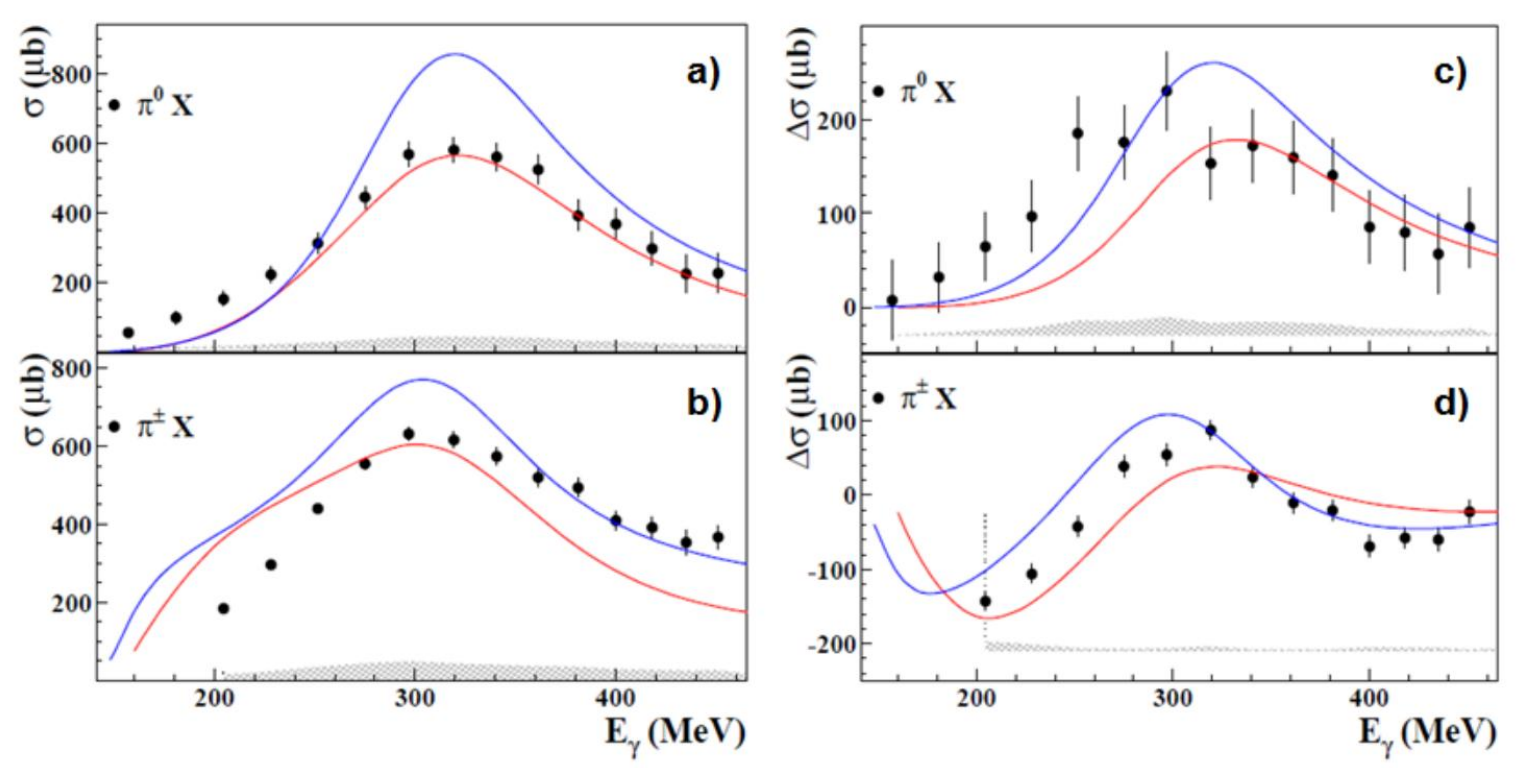

Figure 3: Unpolarised (left) and polarised (right) total cross section for $\gamma^{3} \mathrm{He} \rightarrow \pi^{0} X$ (a and c) and $\gamma^{3} H e \rightarrow \pi^{ \pm} X$ (b and d) channels. The error bars are statistical and the hatched bands show the systematic uncertainties. The measured cross sections are compared to the FA model (red line) and to our PWIA model (blue line).

nels $\gamma^{3} \mathrm{He} \rightarrow \pi^{0} X$ and $\gamma^{3} \mathrm{He} \rightarrow \pi^{ \pm} X$ in the energy region $200<\mathrm{E}_{\gamma}<500 \mathrm{MeV}$ [12]. Here the data are compared to the prediction of the Fix-Arenhövel (FA) model (red line), which is an extension of their deuteron model [13]. The blue line represents a simple plane-wave impulse approximation (PWIA) model [14], where the cross sections are evaluated as an incoherent sum of quasi-free single nucleon contributions, determined using the MAID multipole analysis and the momentum distribution of the nucleons inside ${ }^{3} \mathrm{He}$ as parametrised in [14]. The difference between the two models, i.e. the role of nuclear effects, results in damping and broadening the peak corresponding to the $\Delta$ resonance excitation. Furthermore, to understand the origin of the observed discrepancies between the experimental models and the data, an analysis of both the unpolarised and polarised differential cross sections for the $\gamma^{3} \mathrm{He} \rightarrow \pi^{0} X$ and $\gamma^{3} \mathrm{He} \rightarrow \pi^{ \pm} X$ has been performed: the results can be found in [15].

\section{Conclusions}

Double polarised pion photoproduction experiments are a useful tool for the understanding of the nucleon excitation spectra. The A2 Collaboration at MAMI has performed the measurement of the double polarisation observables $\mathrm{E}$ on deuteron. The experimental data obtained from these experiments can be used also to study the Gerasimov-Drell-Hearn sum rule on neutron. In a complementary way, also polarised ${ }^{3} \mathrm{He}$ targets were used. The new precise results on doublepolarisation measurements of the total and differential cross sections for the partial $\gamma N \rightarrow \pi X$ channels are compared to the existing model predictions and to the few available results. These 
new data significantly increase the available statistics, providing an important testing ground for all existing models. These unprecedented data have proved the feasibility of the use of polarised ${ }^{3} \mathrm{He}$ gas target to check the GDH sum rule on the neutron.

The authors acknowledge support from the Collaborative Research Center (CRC) 1044.

\section{References}

[1] B. Krusche, S. Schadmand, Study of Non-Strange Baryon Resonances with Meson Photoproduction, arXiv:nuclex/ 0306023vl (2003).

[2] I.S. Barker, A. Donnachie, J.K. Storrow, Complete Experiments in Pseudoscalar Photoproduction, Nucl. Phys. B95, 347-356 (1975).

[3] W.-T. Chiang and F. Tabakin, Completeness rules for spin observables in pseudoscalar meson photoproduction, Phys. Rev. C 55, 2054 (1997).

[4] S. B. Gerasimov, A Sum rule for magnetic moments and the damping of the nucleon magnetic moment in nuclei, Sov. J. Nucl. Phys. 2, 430 (1966).

[5] D. Drell and A. C. Hearn, Exact Sum Rule for Nucleon Magnetic Moments, Phys. Rev. Lett. 16, 908 (1966).

[6] D. G. Crabb, W. Meyer, Solid polarized target for nuclear and particle physics experiments, Annu. Rev. Nucl. Part. Sci. 47, 67 (1997).

[7] S. Goertz et al, Highest polarizations in deuterated compounds, Nucl. Instr. Meth. A 526, 43 (2004).

[8] F. Colegrove, Polarization of He $e^{3}$ Gas by Optical Pumping, Phys. Rev. Lett. 132, 2561 (1963).

[9] J. Ahrens et al., Helicity dependence of the total inclusive cross section on the deuteron, Phys. Lett. B 672, 328 (2009).

[10] D. Drechsel, S. Kamalov and L. Tiator, Unitary isobar model MAID2007, Eur. Phys. A 34, 69, (2007).

[11] M. Dieterle, L. Witthauer, F. Cividini et al., First measurement of the polarization observable E and helicity-dependent cross sections in single $\pi^{0}$ photoproduction from quasi-free nucleons, Phys. Lett. $B$ 770, 523 (2017).

[12] P. Aguar Bartolomè et al., Helicity dependence of the total inclusive cross section on the deuteron, Phys. Lett. B 723, $71-77$ (2013).

[13] H. Arenhövel and A. Fix, Incoherent pion photoproduction on the deuteron with polarization observables., Phys. Rev. C 72, 062004 (2005).

[14] R. Schiavilla, C. Pandharipande., Momentum distributions in $A=3$ and 4 nuclei, Nucl. Phys. A 449, 219 (1986).

[15] S. Costanza et al., Helicity dependence of the $\gamma^{3} \mathrm{He} \rightarrow \pi X$ in the $\Delta$ (1232) resonance region, Eur. Phys. J. A 50, 173 (2014). 